\title{
Evolution of minimal access breast surgery
}

\author{
Chi Wei Mok ${ }^{1,2}$, Hung-Wen Lai ${ }^{3,4,5,6,7,8,9,10,11,12}$ \\ ${ }^{1}$ Division of Breast Surgery, Department of Surgery, Changi General Hospital, Singapore, Singapore; ${ }^{2}$ Singhealth Duke-NUS Breast Centre, \\ Singapore, Singapore; ${ }^{3}$ Endoscopy \& Oncoplastic Breast Surgery Center, ${ }^{4}$ Division of General Surgery, ${ }^{5}$ Comprehensive Breast Cancer Center, \\ ${ }^{6}$ School of Medicine, National Yang Ming University, Taipei; ${ }^{7}$ Division of Breast Surgery, Yuanlin Christian Hospital, Yuanlin; ${ }^{8}$ Minimal Invasive \\ Surgery Research Center, Changhua Christian Hospital, Changhua; ${ }^{9}$ Kaohsiung Medical University, Kaohsiung; ${ }^{10}$ School of Medicine, Chung Shan \\ Medical University, Taichung; ${ }^{11}$ Chang Gung University College of Medicine, Taoyuan City; ${ }^{12}$ Division of General Surgery, Kaohsiung Chang Gung \\ Memorial Hospital, Kaohsiung \\ Contributions: (I) Conception and design: HW Lai; (II) Administrative support: None; (III) Provision of study materials or patients: None; (IV) \\ Collection and assembly of data: None; (V) Data analysis and interpretation: CW Mok; (VI) Manuscript writing: All authors; (VII) Final approval of \\ manuscript: All authors. \\ Correspondence to: Dr. Hung-Wen Lai. Endoscopic and Oncoplastic Breast Surgery Center, Changhua Christian Hospital, 135 Nanxiao Street, \\ Changhua 500, Taiwan. Email: hwlai650420@yahoo.com.tw; 143809@cch.org.tw.
}

\begin{abstract}
Surgical management of breast cancer has been evolving rapidly over the past 20-30 years. Prior to this, conventional surgical options were limited to either a mastectomy or breast conserving surgery. The demand for better aesthetic outcomes had driven the development of oncoplastic breast conserving surgery where glandular rearrangement or replacement coupled with thoughtfully placed incisions became the standard approach to breast conserving surgery. As breast surgeons and patients demand for improved aesthetic outcomes, minimally invasive or minimal access breast surgery has gained much attention over the past two decades, from endoscopic assisted to robotic-assisted breast surgery more recently. However, there has been a lack of review articles discussing this relatively recent but under-reported subset of surgical techniques in the management of breast cancer. This article aims to discuss the concept and development of minimal access breast surgery along with a review of current literature on its indications, techniques and outcome measures as well as a discussion on the strengths, limitations as well as future directions. Continued improvement in techniques and advancement of technology will definitely increase the likelihood of minimal access techniques being placed as the standard of care in the management of breast cancer.
\end{abstract}

Keywords: Minimal access; minimally invasive; robotic; endoscopic; breast surgery

Submitted Jul 14, 2019. Accepted for publication Nov 04, 2019.

doi: $10.21037 /$ gs.2019.11.16

View this article at: http://dx.doi.org/10.21037/gs.2019.11.16

\section{Introduction}

Minimally invasive breast surgery, a term coined over the past 2 decades or so essentially described surgical techniques performed with the assistance of endoscopic instruments and more recently robotic surgical platform. Key features of this technique revolved around adequate yet small incision(s) placed in inconspicuous or hidden areas leading to better aesthetic outcomes, while not compromising on safety and allowing for immediate breast reconstruction to be performed through the same incision(s) (1-3). The use of endoscopic instruments or robotic surgical platform help to improve visualization through better optics and thereby allowing for oncologic resection to take place through small incisions (2,4-6). Endoscopic-assisted mastectomy was first performed and popularized in a few Asian countries $(1,5,7-10)$, where the obvious advantage seemed to be better aesthetic outcomes for women with small breasts in whom a breast conserving surgery resulted in poor aesthetic outcomes as well as the risk of inadequate resection or margin involvement. Endoscopic-assisted breast conserving surgery (E-BCS) (11-16) was also subsequently performed 
with glandular rearrangement or level I oncoplastic techniques. There were multiple studies reporting on the technical feasibility, aesthetic and safety outcomes of the technique over the years but it has yet to become the mainstream or standard in the surgical management of breast cancer (1,5,7-16). Possible reason for this includes the lack of long-term follow-up data to establish oncologic safety in terms of loco-regional and distant recurrence as well as disease survival outcomes data. In addition, patient selection and suitability may be another factor on why endoscopic-assisted surgery was not routinely offered and established as standard of care (2,3). More recently, robotic-assisted breast surgery, especially robotic-assisted nipple sparing mastectomy (R-NSM) has gained attention as the possible next step in the evolution of minimally invasive breast surgery (17). So far, there have been a few case series reporting on the technical feasibility, safety and early oncologic outcomes of robotic-assisted mastectomy in a few centers worldwide (18-22). The results were promising and encouraging, however, a recent US FDA safety communication (23) seemed to be casting a doubt and raising a need for closer scrutiny as well as evaluation of this technique to ensure oncological safety are not compromised with the use of this technique in the management of breast cancer. This review article strives to discuss the concept and development of minimal access breast surgery along with a review of current literature on its indications, techniques and outcome measures as well as a discussion on the strengths, limitations as well as future directions that could possibly place minimal access breast surgery as a standard of care in the surgical management of breast cancer.

\section{Discussion}

\section{The concept of minimally invasive versus minimal access breast surgery}

The term 'minimally invasive' has been used to describe endoscopic- and robotic-assisted breast surgery as well as other surgical procedures throughout the years (24). However, the authors suggest that the term 'minimal access' be used in place of 'minimally invasive' in the context of breast surgery for two main reasons: firstly, the incision or access is smaller or hidden if compared to conventional approach; secondly, the dissection and disruption of breast parenchyma are often more extensive in endoscopic- or robotic-assisted breast conserving surgery if compared with conventional methods. The reason lies in the placement of aesthetically pleasing incisions far from the area of interest hence resulting in more extensive dissection required for oncologic resection to take place. For example, endoscopicassisted wide excision of a tumour in the lower inner quadrant through an axillary incision will require dissection of skin flap as well as breast parenchyma from axilla towards the lower inner quadrant whereby a conventional approach will only entail a peri-areolar incision and shorter dissection towards the tumour hence resulting in lesser tissue disruption and trauma.

\section{Minimal access breast surgery: what it truly stands for?}

In line with the concept of minimal access breast surgery, the authors suggest that there are two main broad categories of surgical techniques with or without the use of endoscopic instruments (Figure 1). Under non-endoscopic group, there are various techniques which can be employed and that include but not limited to moving window and retraction with light handle retractors. Endoscopic-assisted breast surgery (EABS) can be further divided into roboticassisted and endoscopic-assisted non-robotic techniques. Endoscopic-assisted non-robotic techniques can be further subdivided according to variations in instruments used such as the use of retraction or insufflation system, single versus multiple ports, use of 3-dimensional (3D) or $4 \mathrm{~K}$ resolution system.

\section{History \& development of endoscopic (non-robotic assisted) and robotic-assisted breast surgery}

For the ease of reference to previous studies, endoscopicassisted non-robotic breast surgery will be interchangeably used with the more familiar terms of E-BCS or endoscopicassisted nipple sparing mastectomy (E-NSM). First case series of E-BCS and E-NSM were reported in 2001 (1) and 2002 (25) respectively, with both studies demonstrating feasibility and satisfactory aesthetic outcomes. Over the next 2 decades, there were altogether 28 studies on EABS (26) with 15 studies on E-BCS (27) and another 14 studies on E-NSM (28-34) (Table 1). Cohort studies comparing conventional and E-BCS or E-NSM showed comparable surgical and short or medium-term oncologic outcomes (Tables 2,3).

R-NSM, on the other hand was first reported in 2015 by Toesca et al. (35) whereby a case series of 3 patients who were BRCA mutation carries received risk reducing mastectomy (RRM) and the authors concluded that the 


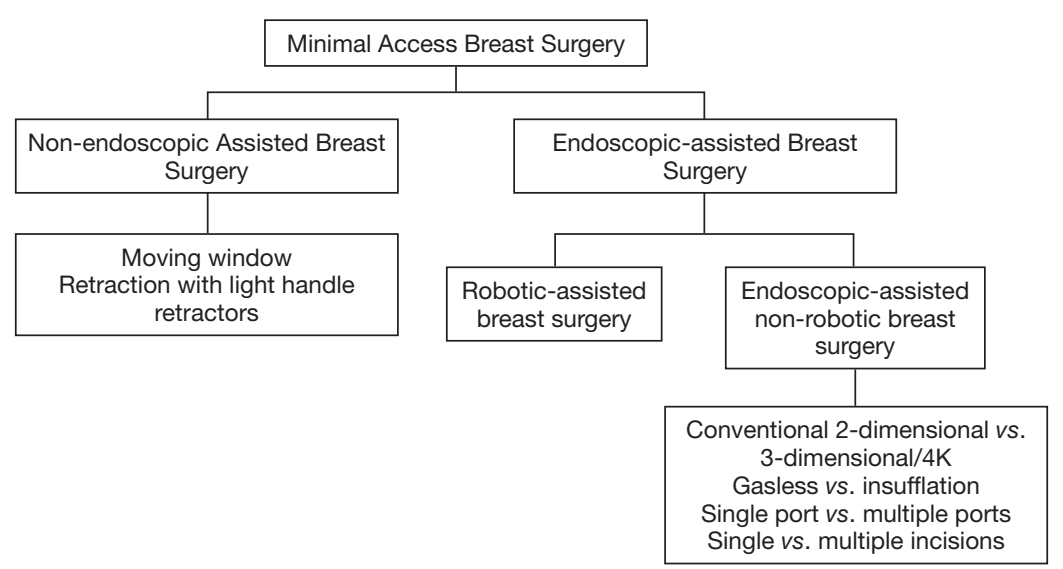

Figure 1 Flowchart of minimal access breast surgery.

Table 1 Summary of studies

\begin{tabular}{|c|c|}
\hline Surgical techniques & Number of studies \\
\hline $\begin{array}{l}\text { Endoscopic-assisted breast conserving } \\
\text { surgery alone }\end{array}$ & 9 \\
\hline $\begin{array}{l}\text { Endoscopic-assisted breast conserving } \\
\text { surgery vs. conventional breast conserving } \\
\text { surgery }\end{array}$ & $6^{*}$ \\
\hline Endoscopic-assisted mastectomy alone & 8 \\
\hline $\begin{array}{l}\text { Endoscopic-assisted mastectomy vs. } \\
\text { conventional mastectomy }\end{array}$ & $4^{*}$ \\
\hline $\begin{array}{l}\text { Endoscopic-assisted mastectomy vs. } \\
\text { conventional breast conserving surgery }\end{array}$ & 2 \\
\hline Total number of studies included & 28 \\
\hline \multicolumn{2}{|c|}{$\begin{array}{l}\text { *, one study compared both E-NSM/C-NSM and E-BCS/C-BCS. } \\
\text { E-NSM, endoscopic assisted nipple sparing mastectomy; } \\
\text { C-NSM, conventional nipple sparing mastectomy; E-BCS, } \\
\text { endoscopic-assisted breast conserving surgery; C-BCS, } \\
\text { conventional breast conserving surgery. }\end{array}$} \\
\hline
\end{tabular}

technique resulted in a feasible and safe operation with better aesthetic outcomes. Over the course of next few years, there were altogether 4 case series (Table 4) reporting on respective institutional experience in the development of R-NSM (18-20,22) with authors from one of the institutions reporting on the learning curve evaluation of this technique in a separate study (21). The advantages reported in all 4 series were similar and that include better visualization with $3 \mathrm{D}$ optics and improved ergonomics from instruments with high degree of freedom of movement. The three main disadvantages of this technique were attributed to prolonged operative time, increased cost as well as availability of robotic surgical platform.

\section{Current evidence on EABS in terms of indications, techniques and outcome measures}

\section{Endoscopic-assisted non-robotic breast surgery}

Indications for E-BCS mainly consisted of early breast cancer with no evidence of multiple lymph node metastasis, skin or chest wall invasion and similar for E-NSM except that no invasion of nipple areolar complex (NAC) as well as multicentric or multifocal cancer were additional indications suitable for E-NSM. Over the years, changes in techniques such as the use of insufflation in place of retraction for improved visualization and sparing of periareolar incision with a change towards a single axillary incision E-NSM resulting in improved surgical outcomes from reduction of NAC necrosis were just two of the many technical improvements made.

Studies conducted over the years have shown the technical feasibility of EABS in achieving equivalent surgical outcomes to conventional surgery. As discussed earlier, the increased operative time in EABS did not result in increased complications and could be reduced after overcoming initial learning curve. There was no clinically or statistically significant increase in terms of intra-operative blood loss. Common complications associated with EABS were similar to conventional techniques and include skin flap or nipple necrosis and this could be attributed to the skin flap thickness and therefore blood supply to the flap especially in cases where the dermis was exposed. Studies using tumescent for skin flap reported ease of dissection and maintenance of 


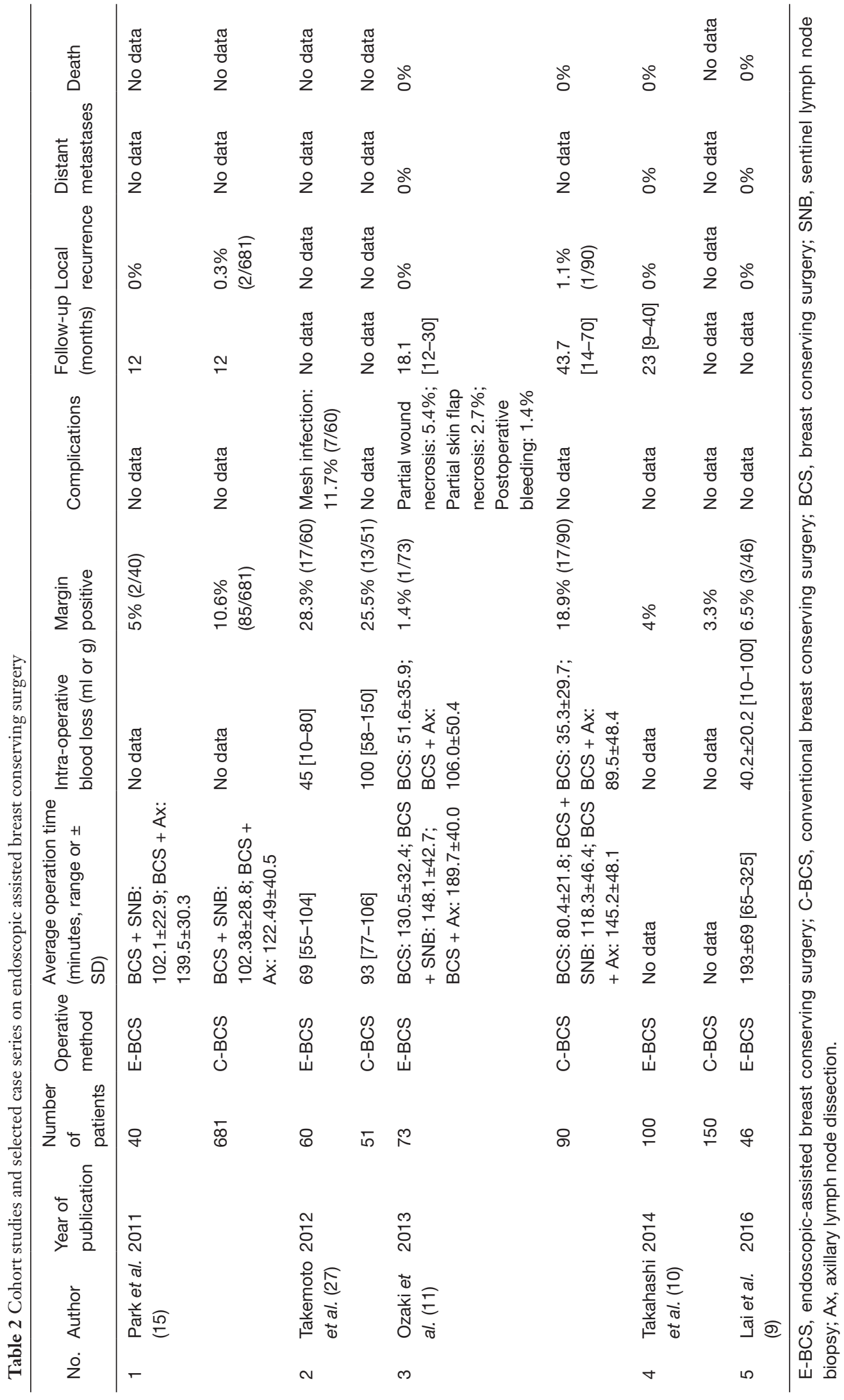




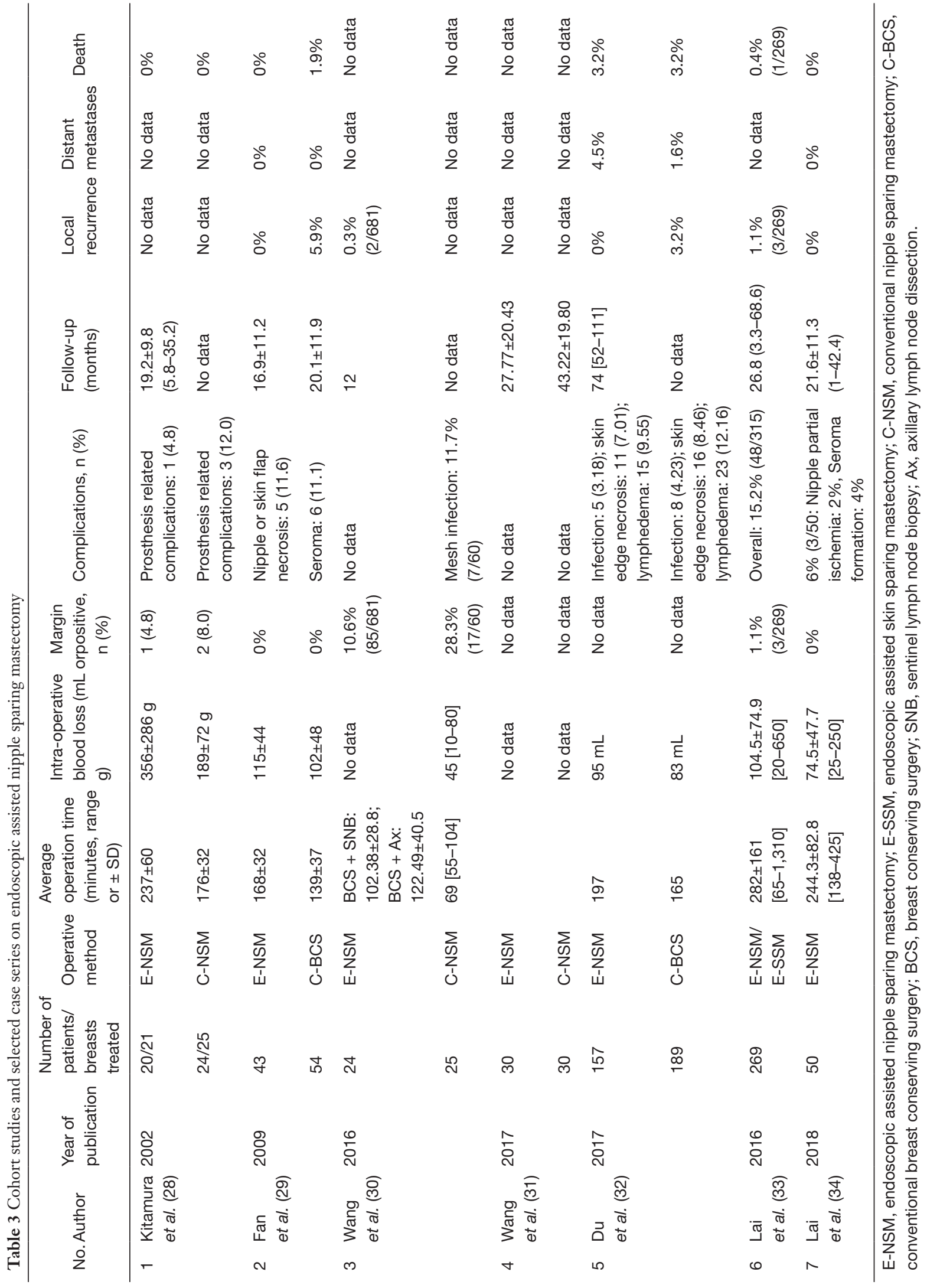




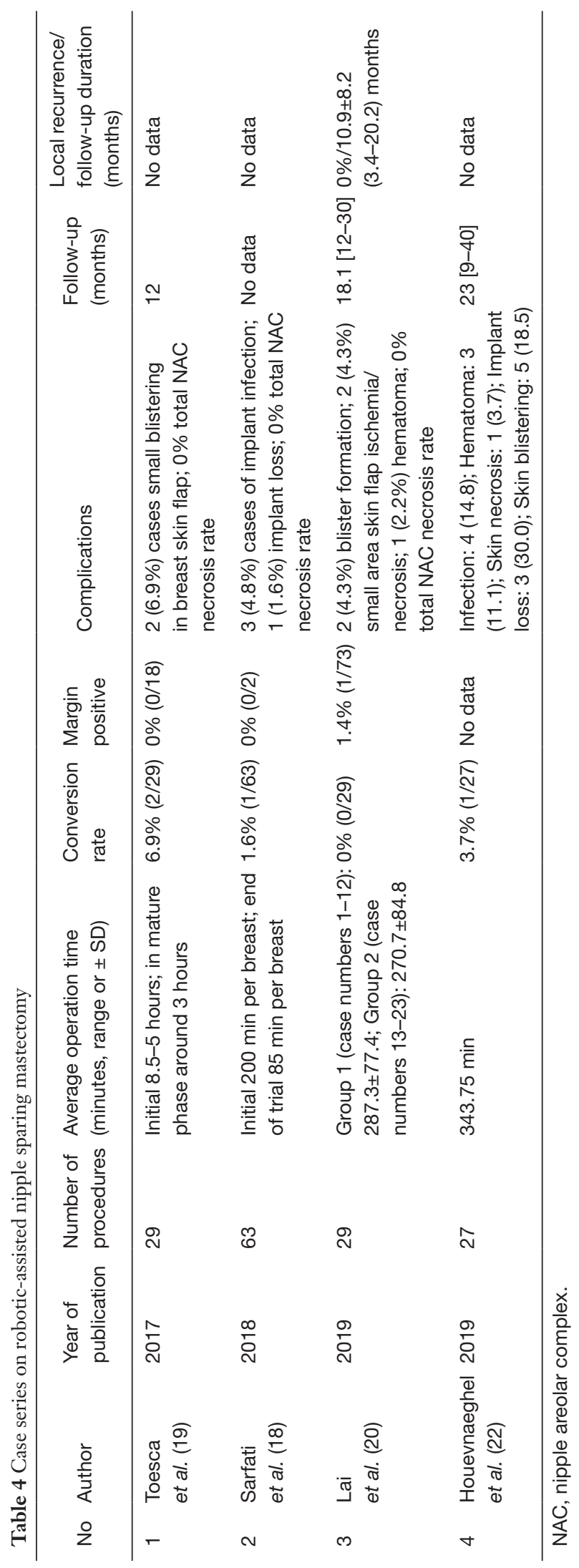

adequate skin flap thickness. Nipple necrosis were mostly reported in cases whereby peri-areolar incision was used and studies with sparing of peri-areolar incision reported lower rate of nipple necrosis. One particular complication of concern would be the high rate of wound infection reported in two studies with the use of absorbable synthetic material or meshes as a volume replacement method in E-BCS. Even though the authors reported subsequent preventive measures such as the use of peri-operative prophylactic antibiotics and frequent changing of surgical gloves, the incidence of infection has not been completely eradicated and the use of these materials should be observed with caution.

In terms of aesthetic outcomes and patient satisfaction assessment, most patients were regarded as being satisfied with the cosmesis especially in terms of scar placement and length of skin incision. However, most of the assessments were done at about 3-6 months after operation and perhaps a repeat assessment should be performed at approximately 2-3 years after the initial operation such that remodeling of breast parenchyma would have been completed and also in cases where adjuvant radiotherapy is required. Details on each of the studies especially with regards to recurrence, conversion or complications rate had previously been reported in a recent review article (26).

\section{Robotic-assisted breast surgery}

Indications of R-NSM as reported in 4 case series were early breast cancer, tumor less than $5 \mathrm{~cm}$ with no evidence of skin, chest wall or NAC involvement. The 4 case series were slightly different from one another with one reporting on prophylactic R-NSM with pre-pectoral placement of implant (18), while another one with patient-reported aesthetic outcomes (20) and the latest one investigating the effects of different techniques of skin flap dissection on complications (22). Incisions used in R-NSM were mainly in the axilla or along the anterior axillary line at the NAC level with incision length ranging from $2.5-5 \mathrm{~cm}$, depending on the breast or specimen size to be removed. One study (20) reported the use of methylene blue in marking the boundaries of breast parenchyma to aid in the dissection. Skin flap dissection were either performed with subcutaneous tunneling technique (20) or sharp dissection with Metzenbaum scissors (18). In terms of outcome measures, R-NSM was proven to be feasible and safe in all studies with low conversion rate, reasonable learning curve (21) and low complications rate. Of notable mention was the $0 \%$ NAC necrosis rate across all studies $(18-20,22)$ 

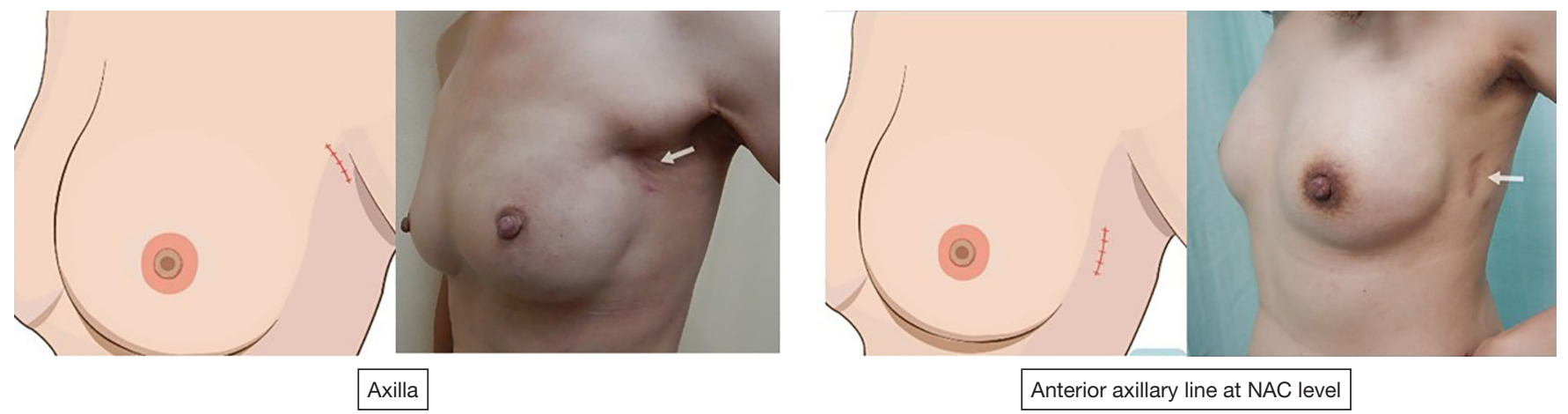

Figure 2 Common incisions used in endoscopic- and robotic-assisted nipple sparing mastectomy.

in which the authors of one study (20) attributed the low NAC necrosis rate to placement of incision far from the $\mathrm{NAC}$ and better visualization with $3 \mathrm{D}$ optics on a robotic surgical platform. Oncologic outcomes were not reported as the follow-up duration was relatively short in all studies. Excellent aesthetic outcomes were reported in all studies and in addition, one study described patient-reported satisfaction rate in which majority of patients were satisfied with the operation especially with regards to placement and length of incision (20).

\section{Advantages and disadvantages of EABS}

\section{Endoscopic-assisted non-robotic breast surgery}

E-BCS or E-NSM allows for better incision or scar placement in inconspicuous areas thereby leading to better cosmesis. Secondly, it allows for tumour resection with adequate margins and hence no compromise on shortand medium-term oncological outcomes. Thirdly, it offers better visualization with the aid of light handle retractors and allows for better precision when it comes to wide excision.

On the other hand, it has its inherent disadvantages as more equipment is required with additional time in the setup and conduct of the operation. This leads to increased operative time if compared to conventional surgery. However, cohort studies have shown that the operative time could be reduced after overcoming initial learning curve and there was no increased risks of complications and adverse outcomes as a result of the longer operative time. Increased cost is also another possible disadvantage as there are disposable instruments used in the conduct of the operation compared to conventional surgery. The solution to this may be the use of re-usable equipment but it would be of great value to have a cost-effectiveness analysis looking at the average cost of E-BCS or E-NSM in the long run.

\section{Robotic-assisted breast surgery}

Robotic surgical platform offered many advantages including better visualization with $3 \mathrm{D}$ optics, improved ergonomics from instruments equipped with a high degree of freedom of movement and better working space due to insufflation used. However, in the authors' experience, robotic surgical platform is most suitable in R-NSM compared to other types of breast surgery. As the incision was placed in the axilla or along the anterior axillary line, aesthetic outcomes were also better if compared to conventional methods (Figure 2). The three main disadvantages of this technique were attributed to prolonged operative time, increased cost as well as availability of robotic surgical platform.

\section{The future and latest advancements in minimal access breast surgery}

The future of minimal access breast surgery is limitless. As shown in Figure 1, variation of techniques can be applied in the conduct of minimal access surgery. Technique that will remain competitive and potentially develop into a standard approach should fulfill 4 criteria in terms of safety (surgical and oncological safety), efficacy, acceptable operative time and cost-effectiveness. Endoscopic-assisted surgery, be it robotic or non-robotic approach has equal chance to develop into standard approach for the surgical management of breast cancer.

\section{Endoscopic-assisted non-robotic breast surgery}

The latest advancements and improvements in non-robotic endoscopic breast surgery would be the use of insufflation as 
well as $3 \mathrm{D}$ or $4 \mathrm{~K}$ endoscopic system. The use of insufflation has been reported to result in better working space as well as improved hemostasis possibly due to positive pressure from the insufflation (34). The preliminary experience of the use of single port insufflation 3D endoscopic system in E-NSM was just recently reported as a new technique (36) and the authors demonstrated safety, feasibility as well as excellent aesthetic outcomes. Other than 3D system, there are also $4 \mathrm{~K}$ high definition (HD) system in the market which could potentially improve visualization further and be used in E-NSM with insufflation.

\section{Robotic-assisted breast surgery}

Da Vinci robotic surgical platform is at the forefront of R-NSM. Da Vinci Si and Xi system were used in current reported series. New robotic surgical platform, da Vinci SP or single port system is the latest addition to the da Vinci ${ }^{\circledR}$ Surgical System (Intuitive Surgical, Inc., Sunnyvale, CA, USA). In what was described as the first platform with fully wristed and elbowed instruments within a single port system through a $2.5 \mathrm{~cm}$ cannula, better versatility and thereby reach would be very much anticipated. However, the size of the cannula at $2.5 \mathrm{~cm}$ might pose significant challenge as incision may need to be extended at the end of operation for specimen extraction and this may render the small cannula size irrelevant. As the system is currently available in only selected countries or institutions worldwide, the authors would foresee increased use of the system in R-NSM in years to come. In addition, there are other robotic surgical platforms in the market offering different niche areas of expertise and the potential of their use in breast surgery are limitless (37).

\section{International endoscopic and robotic breast surgery symposium (IERBS) 2019}

In the recently concluded IERBS from $24^{\text {th }}$ to $25^{\text {th }}$ May 2019 in Taiwan, pioneers and experts in the field of endoscopic and robotic breast surgery came together and shared their respective institutional experience in the first conference dedicated to endoscopic and robotic-assisted breast surgery. Sharing of experience and technical know-how are of utmost importance in promoting the development of endoscopic breast surgery. In addition, experts in the field of R-NSM also came together and developed the first IERBS consensus statement on robotic mastectomy which covered 6 domains including indications, contraindications, technical considerations, patient counselling, outcome measures as well as training and learning curve assessment. The consensus statement will be published in the near future, providing experts' opinion and guidance in the fastdeveloping field of robotic-assisted breast surgery.

\section{Conclusions}

The era of minimal access breast surgery is already here and will most likely stay for the next decade or so. Continued improvement in techniques and advancement of technology will definitely increase the likelihood of minimal access techniques being placed as the standard of care in the management of breast cancer. Future directions in terms of international multicenter collaborations and a structured training system for endoscopic and/or robotic-assisted breast surgery are of paramount importance while the long-term oncologic outcomes as well as cost-effectiveness analyses are much anticipated in the near future to further consolidate the use of EABS in the surgical management of breast cancer.

\section{Acknowledgments}

The authors would like to thank Yun-Ting Chang for the graphic illustrations used in this article.

Funding: This study was funded by the Ministry of Science and Technology of Taiwan, MOST 108-2314-B-371-006-. This study was also sponsored by research funding provided by Changhua Christian Hospital 108-CCH-IRP-122.

\section{Footnote}

Conflicts of Interest: Dr. HW Lai was the organizing chair and founder of IERBS 2019. Dr. CW Mok was the secretary general of IERBS 2019.

Ethical Statement: The authors are accountable for all aspects of the work in ensuring that questions related to the accuracy or integrity of any part of the work are appropriately investigated and resolved.

\section{References}

1. Tamaki Y, Sakita I, Miyoshi Y, et al. Transareolar endoscopy-assisted partial mastectomy: a preliminary report of six cases. Surg Laparosc Endosc Percutan Tech 2001;11:356-62.

2. Leff DR, Vashisht R, Yongue G, et al. Endoscopic breast 
surgery: where are we now and what might the future hold for video-assisted breast surgery? Breast Cancer Res Treat 2011;125:607-25.

3. Ozaki S, Ohara M. Endoscopy-assisted breastconserving surgery for breast cancer patients. Gland Surg 2014;3:94-108.

4. Fukuma E. Endoscopic breast surgery for breast cancer. Nihon Geka Gakkai Zasshi 2006;107:64-8.

5. Tukenmez M, Ozden BC, Agcaoglu O, et al. Videoendoscopic single-port nipple-sparing mastectomy and immediate reconstruction. J Laparoendosc Adv Surg Tech A 2014;24:77-82.

6. Lai HW, Chen ST, Lin SL, et al. Technique for single axillary incision robotic assisted quadrantectomy and immediate partial breast reconstruction with robotic latissimus dorsi flap harvest for breast cancer: A case report. Medicine (Baltimore) 2018;97:e11373.

7. Sakamoto N, Fukuma E, Higa K, et al. Early results of an endoscopic nipple-sparing mastectomy for breast cancer. Indian J Surg Oncol 2010;1:232-9.

8. Ito K, Kanai T, Gomi K, et al. Endoscopic-assisted skinsparing mastectomy combined with sentinel node biopsy. ANZ J Surg 2008;78:894-8.

9. Lai HW, Chen ST, Chen DR, et al. Current Trends in and Indications for Endoscopy-Assisted Breast Surgery for Breast Cancer: Results from a Six-Year Study Conducted by the Taiwan Endoscopic Breast Surgery Cooperative Group. PLoS One 2016;11:e0150310.

10. Takahashi H, Fujii T, Nakagawa S, et al. Usefulness of endoscopic breast-conserving surgery for breast cancer. Surg Today 2014;44:2037-44.

11. Ozaki S, Ohara M, Shigematsu H, et al. Technical feasibility and cosmetic advantage of hybrid endoscopyassisted breast-conserving surgery for breast cancer patients. J Laparoendosc Adv Surg Tech A 2013;23:91-9.

12. Nakajima H, Fujiwara I, Mizuta N, et al. Video-assisted skin-sparing breast-conserving surgery for breast cancer and immediate reconstruction with autologous tissue: clinical outcomes. Ann Surg Oncol 2009;16:1982-9.

13. Hong YI, Shin H. Endoscopy-assisted breast conserving surgery for breast cancer: A preliminary clinical experience. J Breast Cancer 2010;13:138-46.

14. Owaki T, Yoshinaka H, Ehi K, et al. Endoscopic quadrantectomy for breast cancer with sentinel lymph node navigation via a small axillary incision. Breast 2005;14:57-60.

15. Park HS, Lee JS, Lee JS, et al. The feasibility of endoscopy-assisted breast conservation surgery for patients with early breast cancer. J Breast Cancer 2011;14:52-7.

16. Saimura M, Mitsuyama S, Anan K, et al. Endoscopyassisted breast-conserving surgery for early breast cancer. Asian J Endosc Surg 2013;6:203-8.

17. Selber JC. Robotic Nipple-Sparing Mastectomy: The Next Step in the Evolution of Minimally Invasive Breast Surgery. Ann Surg Oncol 2019;26:10-1.

18. Sarfati B, Struk S, Leymarie N, et al. Robotic Prophylactic Nipple-Sparing Mastectomy with Immediate Prosthetic Breast Reconstruction: A Prospective Study. Ann Surg Oncol 2018;25:2579-86.

19. Toesca A, Peradze N, Manconi A, et al. Robotic nipplesparing mastectomy for the treatment of breast cancer: Feasibility and safety study. Breast 2017;31:51-6.

20. Lai HW. Robotic Nipple-Sparing Mastectomy and Immediate Breast Reconstruction with Gel Implant. Ann Surg Oncol 2019;26:53-4.

21. Lai HW, Wang CC, Lai YC, et al. The learning curve of robotic nipple sparing mastectomy for breast cancer: An analysis of consecutive 39 procedures with cumulative sum plot. Eur J Surg Oncol 2019;45:125-33.

22. Houvenaeghel G, Bannier M, Rua S, et al. Breast cancer robotic nipple sparing mastectomy: evaluation of several surgical procedures and learning curve. World J Surg Oncol 2019;17:27.

23. US Food and Drug Administration (FDA). Caution When Using Robotically-Assisted Surgical Devices in Women's Health including Mastectomy and Other Cancer-Related Surgeries: FDA Safety Communication February 2019 [Internet]. Available online: https://www.fda.gov/medicaldevices/safety-communications/caution-when-usingrobotically-assisted-surgical-devices-womens-healthincluding-mastectomy-and?utm_campaign=RoboticallyAssisted Surgical Devices in Women\%27s Health\&utm_ medium=email\&utm_source=El

24. Mack MJ. Minimally invasive and robotic surgery. JAMA 2001;285:568-72.

25. Nakajima H, Sakaguchi K, Mizuta N, et al. Video-assisted total glandectomy and immediate reconstruction for breast cancer. Biomed Pharmacother 2002;56 Suppl 1:205s-8s.

26. Mok CW, Lai HW. Endoscopic-assisted surgery in the management of breast cancer: 20 years review of trend, techniques and outcomes. Breast 2019;46:144-56.

27. Takemoto N, Koyanagi A, Yamamoto H. Comparison between endoscope-assisted partial mastectomy with filling of dead space using absorbable mesh and conventional conservative method on cosmetic outcome in patients with stage I or II breast cancer. Surg Laparosc Endosc Percutan 
Tech 2012;22:68-72.

28. Kitamura K, Ishida M, Inoue H, et al. Early results of an endoscope-assisted subcutaneous mastectomy and reconstruction for breast cancer. Surgery 2002;131:S324-9.

29. Fan LJ, Jiang J, Yang XH, et al. A prospective study comparing endoscopic subcutaneous mastectomy plus immediate reconstruction with implants and breast conserving surgery for breast cancer. Chin Med J (Engl) 2009; 122:2945-50.

30. Wang ZH, Qu X, Teng CS, et al. Preliminary results for treatment of early stage breast cancer with endoscopic subcutaneous mastectomy combined with endoscopic sentinel lymph node biopsy in China. J Surg Oncol 2016;113:616-20.

31. Wang Y, Wu JX, Guan S. A Technique of Endoscopic Nipple-Sparing Mastectomy for Breast Cancer. JSLS 2017. doi: 10.4293/JSLS.2017.00028.

32. Du J, Liang Q, Qi X, et al. Endoscopic nipple sparing mastectomy with immediate implant-based reconstruction versus breast conserving surgery: a long-term study. Sci Rep 2017;7:45636.

Cite this article as: Mok CW, Lai HW. Evolution of minimal access breast surgery. Gland Surg 2019;8(6):784-793. doi: 10.21037 /gs.2019.11.16
33. Lai HW, Chen ST, Chen DR, et al. Current Trends in and Indications for Endoscopy-Assisted Breast Surgery for Breast Cancer: Results from a Six-Year Study Conducted by the Taiwan Endoscopic Breast Surgery Cooperative Group. PLoS One 2016;11:e0150310.

34. Lai HW, Lin SL, Chen ST, et al. Single-Axillary-Incision Endoscopic-Assisted Hybrid Technique for NippleSparing Mastectomy: Technique, Preliminary Results, and Patient-Reported Cosmetic Outcome from Preliminary 50 Procedures. Ann Surg Oncol 2018;25:1340-9.

35. Toesca A, Manconi A, Peradze N, et al. 1931 Preliminary report of robotic nipple-sparing mastectomy and immediate breast reconstruction with implant. Eur J Cancer 2015;51:S309.

36. Lai HW, Chen ST, Mok CW, et al. Single-port 3-dimensional Videoscope-assisted Endoscopic Nipplesparing Mastectomy in the Management of Breast Cancer. Plast Reconstr Surg Glob Open 2019; 7:e2367.

37. Peters BS, Armijo PR, Krause C, et al. Review of emerging surgical robotic technology. Surg Endosc 2018;32:1636-55. 